\title{
Hundreds of starting points for spliceosomal PPI modulators identified by crystallographic fragment screening with the F2X-Universal Library
}

\author{
T. Barthel ${ }^{1,3}$, J. Wollenhaupt ${ }^{1}$, G. M. A. Lima ${ }^{2}$, M. C. Wahl ${ }^{3}$, M. S. Weiss ${ }^{1}$ \\ ${ }^{1}$ Helmholtz-Zentrum Berlin, Macromolecular Crystallography, Albert-Einstein-Str. 15, 12489 Berlin, Germany, \\ ${ }^{2}$ MAX IV Laboratory, BioMAX, Fotongatan 2, 22484 Lund, Sweden, \\ ${ }^{3}$ Freie Universität Berlin, Institute of Chemistry and Biochemistry, Laboratory of Structural Biochemistry, \\ Takustraße 6, 14195 Berlin, Germany \\ tatjana.barthel@helmholtz-berlin.de
}

\begin{abstract}
Almost all cellular processes depend on protein-protein interactions (PPI) which therefore are promising targets for drug design or tool compound development. PPIs as targets have the advantage that the interaction surfaces are highly specific and unique, meaning potent and selective compounds are less likely to cause side effects compared to targeting enzyme activities. However, targeting PPIs is usually challenging due to their large interaction surface and shallow pockets. It is important for such surfaces to find hotspots where compounds engage in strong interactions with the protein. Finding hotspots can be achieved by screening fragments (organic compounds $<300 \mathrm{Da}$ ), which form specific interactions with the target. These fragments can be used as starting points for compound development. In order to identify fragments bound to a protein, crystallographic fragment screening [1] is a great tool as it not only informs about the presence of a fragment, but also about its 3D position towards the protein. Thus, enabling structure-guided drug design or tool compound development for any target that can be crystallized reproducibly and gives diffraction data to sufficient resolution.
\end{abstract}

We chose the spliceosomal protein-protein complex of yeast Aar2 and Prp8 $8^{\mathrm{RNaseH}}$ (AR) [2], to screen our novel over 100-membered F2X-Universal Library [3]. The spliceosome is a large cellular machine that depends on dynamically changing PPIs throughout its functional cycle and thus is an ideal object of study for PPI compound development. Prp8 is highly conserved in the spliceosome and acts as a scaffolding protein. Prp8 is shuttled into the nucleus via a shuttling factor Aar2. Utilizing the complex for our screen has the advantage to identify fragments bound close to the interface of AR while also probing the surfaces of Prp $8^{\mathrm{RNaseH}}$ and Aar2 at the same time. That means information can be gathered to either develop an enhancer for the interaction of AR or inhibitors for other interactions $\operatorname{Prp} 8^{\mathrm{RNaseH}}$ is involved in or to find potentially interesting sites on Aar2. In previous work a representative subset of the F2X-Universal Library, the F2X-Entry Screen (96 fragments) was screened against AR, which already resulted in 20 unique hits that hinted towards possible hot spots [3]. However, in order to verify the found hotspots as such, to potentially find more hotspots and to confirm the found binding motifs of the fragments the full F2X-Universal Library, i.e. 1013 compounds in total, were screened. For the automatic data analysis FragMAXapp [4] in combination with cluster4x [5] was utilized and this resulted in 278 unique hits scattered across the two proteins highlighting hotspots on their surfaces. This translates into a hit rate of $27,5 \%$, which is the highest hit rate for such a large screen to the best of our knowledge and successfully validated the library. The additional hits found by screening the complete F2X-Universal Library validated certain binding modes presented by the F2X-Entry Screen and provided novel hotspots. This way we gained a large number of interesting starting points to develop potential modulators for several PPIs inside the spliceosome.

[1] Wollenhaupt, J., Barthel, T., Lima, G. M. A., Metz, A., Wallacher, D., Jagudin, E., Huschmann, F. U., Hauß, T., Feiler, C. G., Gerlach, M., Hellmig, M., Förster, R., Steffien, M., Heine, A., Klebe, G., Mueller, U. \& Weiss, M. S. (2021). J. Vis. Exp. 2021, 1-19

[2] Weber, G., Cristão, V. F., Santos, K. F., Jovin, S. M., Heroven, A. C., Holton, N., Lührmann, R., Beggs, J. D. \& Wahl, M. C. (2013). Genes Dev. $27,525-540$.

[3] Wollenhaupt, J., Metz, A., Barthel, T., Lima, G. M. A., Heine, A., Mueller, U., Klebe, G. \& Weiss, M. S. (2020). Structure. 28, 694-706.

[4] Lima, G. M. A., Talibov, V. O., Jagudin, E., Sele, C., Nyblom, M., Knecht, W., Logan, D. T., Sjögren, T. \& Mueller, U. (2020). Acta Crystallogr. Sect. D. 76, 771-777.

[5] Ginn, H. M. (2020). Acta Crystallogr. Sect. D Struct. Biol. 76, 1134-1144.

Keywords: crystallographic fragment screening, spliceosome, protein-protein interaction, structure-guided drug design 